\title{
Endoscopic submucosal dissection for papillary adenocarcinoma of the stomach: low curative resection rate but favorable long-term outcomes after curative resection
}

\author{
Tae-Se Kim ${ }^{1}$ · Byung-Hoon Min ${ }^{1}$ (D $\cdot$ Kyoung-Mee Kim² ${ }^{2}$ Jun Haeng Lee ${ }^{1} \cdot$ Poong-Lyul Rhee ${ }^{1} \cdot$ Jae J. Kim ${ }^{1}$
}

Received: 31 May 2018 / Accepted: 17 July 2018 / Published online: 23 July 2018

(c) The International Gastric Cancer Association and The Japanese Gastric Cancer Association 2018

\begin{abstract}
Background The previous studies demonstrated aggressive clinicopathologic features of papillary early gastric cancer (EGC). This raised concerns about the appropriateness of current Japanese guidelines that recommend the same endoscopic submucosal dissection (ESD) criteria for papillary EGC as for well-differentiated (WD) or moderately differentiated (MD) EGCs. Methods This study included 4140 patients who underwent ESD for differentiated-type EGC ( 87 papillary EGCs and 4259 WD or MD EGCs). The clinicopathologic characteristics and short- and long-term outcomes of ESD for papillary EGC were reviewed and compared with those for WD or MD EGC.

Results Papillary EGCs were larger, and had higher lymphovascular and submucosal invasion rates than WD or MD EGCs. Lateral resection margin involvement and histological heterogeneity were found more frequently in papillary EGC than in WD or MD EGC. En bloc with R0 resection and curative resection rates of papillary EGC were 85.1 and 49.4\%, respectively, and both were significantly lower than those of WD or MD EGC (93.0 and 82.2\%). In mucosal cancers, curative resection rates of papillary EGC and WD or MD EGC were 72.5 and 93.7\%, respectively. Among patients undergoing curative ESD for papillary EGC, no extra-gastric recurrences occurred during median 58 months of follow-up. Metachronous recurrence occurred in $5.2 \%$ of cases.

Conclusions Given the favorable long-term outcomes after curative resection, ESD might be indicated for papillary EGC according to the current Japanese guidelines. As papillary EGC has considerable lymphovascular and submucosal invasion rates, careful histological examination is required to accurately determine whether curative ESD is achieved.
\end{abstract}

Keywords Gastric cancer · Papillary adenocarcinoma $\cdot$ Endoscopic submucosal dissection

\section{Introduction}

In the current Japanese guidelines, papillary adenocarcinoma of the stomach is classified as a differentiated-type cancer, as in well-differentiated (WD) and moderately differentiated (MD) adenocarcinoma. In addition, the guidelines recommend applying the same indications of endoscopic resection (ER) for papillary early gastric cancer (EGC) as

Byung-Hoon Min

bhmin@skku.edu

1 Department of Medicine, Samsung Medical Center, Sungkyunkwan University School of Medicine, 81 Irwon-ro, Gangnam-gu, Seoul 06351, South Korea

2 Department of Pathology and Translational Genomics, Samsung Medical Center, Sungkyunkwan University School of Medicine, Seoul, South Korea for WD or MD EGC [1]. To be appropriately indicated for ER, a tumor should have minimal risk of lymph node (LN) metastasis. However, several Japanese and Korean studies have revealed the aggressive clinicopathologic features of papillary EGC, including high lymphovascular invasion and LN metastasis rates [2-6], thus raising concerns about the appropriateness of the current guidelines. In contrast, our group recently demonstrated that the $\mathrm{LN}$ metastasis rate of papillary EGC was comparable to that of WD or MD EGC, despite the higher lymphovascular invasion rate of papillary EGC [7]. No papillary EGC exhibited LN metastasis if it met the curative ER criteria.

Although our previous study based on large-scale surgical data supported the use of ER for papillary EGC treatment, long-term follow-up data after ER are essential to validate the appropriateness of the current guidelines. To 
date, however, few studies have reported the long-term as well as short-term outcomes of ER for papillary EGC [3].

In the present study, we aimed to elucidate the clinicopathologic features and short-term outcomes of endoscopic submucosal dissection (ESD) for papillary EGC compared to those for WD or MD EGC. In addition, we analyzed the long-term outcomes of ESD for papillary EGC to evaluate the validity of the current guidelines.

\section{Methods}

\section{Patients}

From January 2007 to December 2016, 4156 patients with 4372 differentiated-type EGCs (WD or MD EGCs and papillary EGCs) underwent their first ESD at Samsung Medical Center. Among them, the following patients were excluded from the study population: eight patients (eight EGCs) undergoing ESD for EGC in the remnant stomach, and eight patients with both WD or MD EGC and papillary EGC (six patients with two EGCs and two patients with three EGCs). Finally, 4140 patients with 4346 EGCs (87 papillary EGCs, 4259 WD or MD EGCs) were included for analysis. Clinicopathologic data and outcomes after ESD were obtained through the retrospective review of medical records from the intranet resources of Samsung Medical Center. All enrolled patients provided written informed consent according to our institutional guidelines. The study protocol was approved by the institutional review board of Samsung Medical Center.

\section{ESD procedures and histopathological evaluation}

The ESD procedures of our institution have been described in detail elsewhere $[8,9]$. ESD specimens were stretched, pinned to a polystyrene plate, and totally immersed in $10 \%$ neutral buffered formalin for more than $12 \mathrm{~h}$ for fixation. After fixation, the specimen was sectioned serially at 2-mm intervals, parallel to a line that included the closest resection margin of the specimen, so that both the lateral and vertical margins could be assessed [10].

Papillary adenocarcinoma was defined as a tumor in which more than $50 \%$ of the tumor area contained papillary structures composed of epithelial projections with a central fibrovascular core as a scaffold [11]. Histological heterogeneity was defined when signet ring-cell carcinoma or poorly differentiated adenocarcinoma components co-existed in an ESD specimen as a minor portion (less than $50 \%$ of the tumor area), in accordance with Korean and Japanese guidelines $[1,12]$.

\section{Definitions}

En bloc resection was defined as resection of the tumor in one piece with no endoscopically residual tumor. R0 resection was defined as resection of the tumor without any histological evidence of cancer cells on the lateral and vertical margins. The resection was defined as curative when all the following criteria were fulfilled [1]: well- or moderately differentiated EGC or papillary EGC, en bloc resection, negative lateral resection margins, negative vertical resection margin, and no lymphovascular invasion: (1) tumor size $\leq 2 \mathrm{~cm}$, mucosal cancer, no ulcer in the tumor; (2) tumor size $>2 \mathrm{~cm}$, mucosal cancer, no ulcer in the tumor; (3) tumor size $\leq 3 \mathrm{~cm}$, mucosal cancer, ulcer in the tumor; or (4) tumor size $\leq 3 \mathrm{~cm}$, SM1 cancer (submucosal invasion depth $<500 \mu \mathrm{m}$ from the muscularis mucosa layer).

Local recurrence was defined when cancer was detected at the primary resection site in a follow-up esophagogastroduodenoscopy (EGD) after R0 resection. A synchronous lesion was defined as a cancer detected at a location other than the primary resection site within 12 months after ESD. Metachronous recurrence was defined as a cancer detected at a location other than the primary resection site at least 12 months after ESD. Recurrence was evaluated in patients undergoing ESD from January 2007 to December 2014.

\section{Follow-up after ESD}

EGD along with a biopsy was performed 2 months after ESD to confirm the healing of the artificial ulcer and to exclude the presence of any residual tumor. Thereafter, EGD with a biopsy and abdominal computed tomography (CT) were carried out biannually for 3 years, and annually from the fourth to the fifth year after ESD.

\section{Statistical analysis}

Categorical variables were analyzed using the Chi-square test or Fisher's exact test. Continuous variables were analyzed using Student's $t$ test or the Mann-Whitney test. A $P$ value less than 0.05 was considered statistically significant.

\section{Results}

\section{Clinicopathologic features of papillary EGC versus WD or MD EGC}

The study population included 86 patients with 87 papillary EGCs and 4054 patients with 4259 WD or MD EGCs. Table 1 summarizes the clinicopathologic characteristics 
Table 1 Clinicopathologic characteristics of papillary versus well- or moderately differentiated early gastric cancer

\begin{tabular}{|c|c|c|c|}
\hline & Papillary EGC $(n=87)$ & $\begin{array}{l}\text { WD or MD EGC } \\
(n=4259)\end{array}$ & $P$ value \\
\hline Age $\left(\right.$ years) ${ }^{a}$ & & & 0.047 \\
\hline Mean \pm SD & $65.4 \pm 10.1$ & $63.4 \pm 9.8$ & \\
\hline Median (range) & $66(37-83)$ & $64(27-93)$ & \\
\hline Gender $(\%)^{\mathrm{a}}$ & & & 0.605 \\
\hline Male & $65(75.6)$ & $3159(77.9)$ & \\
\hline Female & $21(24.4)$ & $895(22.1)$ & \\
\hline Tumor site (\%) & & & 0.618 \\
\hline Antrum/angle & $60(69.0)$ & $3072(72.1)$ & \\
\hline Body & $23(26.4)$ & $1061(24.9)$ & \\
\hline Fundus/cardia & $4(4.6)$ & $126(3.0)$ & \\
\hline Macroscopic type (\%) & & & $<0.001$ \\
\hline Elevated & $69(79.3)$ & $2175(51.1)$ & \\
\hline Flat or depressed & $18(20.7)$ & $2084(48.9)$ & \\
\hline Tumor size on pathology $(\mathrm{cm})$ & & & $<0.001$ \\
\hline Mean \pm SD & $2.1 \pm 1.2$ & $1.5 \pm 0.9$ & \\
\hline Median (range) & $1.8(0.2-5.2)$ & $1.4(0.1-11.0)$ & \\
\hline Tumor depth (\%) & & & $<0.001$ \\
\hline Mucosa & $51(58.6)$ & $3258(82.8)$ & \\
\hline SM1 & $12(13.8)$ & $316(7.4)$ & \\
\hline $\mathrm{SM} 2$ or SM3 & $24(27.6)$ & $415(9.7)$ & \\
\hline Lymphatic invasion (\%) & & & $<0.001$ \\
\hline Absent & $65(74.7)$ & $3894(91.4)$ & \\
\hline Present & $22(25.3)$ & $365(8.6)$ & \\
\hline Venous invasion (\%) & & & 0.003 \\
\hline Absent & $82(94.3)$ & $4213(98.9)$ & \\
\hline Present & $5(5.7)$ & $46(1.1)$ & \\
\hline Ulcer $(\%)$ & & & 1.000 \\
\hline Absent & $87(100)$ & $4243(99.6)$ & \\
\hline Present & $0(0.0)$ & $16(0.4)$ & \\
\hline Lateral margin (\%) & & & 0.011 \\
\hline Negative & $78(89.7)$ & $4089(96.0)$ & \\
\hline Positive & $9(10.3)$ & $146(3.4)$ & \\
\hline Undetermined & $0(0.0)$ & $24(0.6)$ & \\
\hline Vertical margin (\%) & & & 1.000 \\
\hline Negative & $85(97.7)$ & $4136(97.1)$ & \\
\hline Positive & $2(2.3)$ & $101(2.4)$ & \\
\hline Undetermined & $0(0.0)$ & $22(0.5)$ & \\
\hline Histologic heterogeneity (\%) & & & 0.033 \\
\hline Absent & $73(83.9)$ & $3862(90.7)$ & \\
\hline Present & $14(16.1)$ & 397 (9.3) & \\
\hline
\end{tabular}

$E G C$ early gastric cancer, $W D$ well differentiated, $M D$ moderately differentiated, $S D$ standard deviation, $S M 1$ submucosal invasion depth $<500 \mu \mathrm{m}$ from muscularis mucosa layer, SM2 or SM3 submucosal invasion depth $\geq 500 \mu \mathrm{m}$ from muscularis mucosa layer

${ }^{\mathrm{a}}$ A total of 86 papillary EGC patients and 4054 WD or MD EGC patients were included of patients with papillary EGC versus WD or MD EGC. Patients with papillary EGC were older and more likely to have macroscopically elevated tumors than patients with WD or MD EGC. Papillary EGCs were larger, and had significantly higher submucosal and lymphovascular invasion rates than WD or MD EGCs. Lateral resection margin involvement and histological heterogeneity were found more frequently in papillary EGC than in WD or MD EGC. In the WD or MD EGC group, patients with lateral margin involvement had a significantly higher rate of histological 
heterogeneity than those without margin involvement (26.7 versus $8.7 \%$ ). In the papillary EGC group, the histological heterogeneity rates in patients with and without lateral margin involvement were 22.2 and $15.4 \%$, respectively, which did not differ significantly.

\section{Short-term outcomes of ESD for papillary EGC versus WD or MD EGC}

Table 2 summarizes the short-term outcomes of ESD for papillary EGC versus WD or MD EGC. The en bloc resection, R0 resection, and en bloc with R0 resection rates for papillary EGC were 94.3, 87.4, and 85.1\%, respectively, which were significantly lower than those for WD or MD EGC. The curative resection rates were $49.4 \%$ for papillary EGC and $82.2 \%$ for WD or MD EGC, and differed significantly between the two groups.

In the present study, the submucosal invasion rate was significantly higher for papillary EGC than for WD or MD EGC, which may have contributed to the worse short-term outcomes of papillary EGC shown in Table 2. Therefore, we further analyzed the short-term outcomes of ESD in mucosal cancers (Table 3). The en bloc with R0 resection rates were 88.2 and $95.6 \%$ in papillary and WD or MD

Table 2 Short-term outcomes of endoscopic submucosal dissection for papillary versus well- or moderately differentiated early gastric cancer

\begin{tabular}{lccc}
\hline & \multicolumn{2}{l}{ Total } & P value \\
\cline { 2 - 3 } & $\begin{array}{l}\text { Papil- } \\
\text { lary EGC } \\
(n=87)\end{array}$ & $\begin{array}{l}\text { WD or } \\
\text { MD EGC } \\
(n=4259)\end{array}$ & \\
& & & 0.027 \\
\hline En bloc resection (\%) & $82(94.3)$ & $4179(98.1)$ & \\
Yes & $5(5.7)$ & $80(1.9)$ & \\
No & & & 0.011 \\
R0 resection (\%) & $76(87.4)$ & $4002(94.0)$ & \\
Yes & $11(12.6)$ & $257(6.0)$ & \\
No or undetermined & & & 0.004 \\
En bloc and R0 resection $(\%)$ & & & \\
Yes & $74(85.1)$ & $3961(93.0)$ & \\
No or undetermined & $13(14.9)$ & $298(7.0)$ & \\
Curative resection (\%) & & & 0.001 \\
Yes & $43(49.4)$ & $3502(82.2)$ & \\
No & $44(50.6)$ & $757(17.8)$ & \\
Perforation (\%) & & & \\
Absent & $84(96.6)$ & $4172(98.0)$ & \\
Present & $3(3.4)$ & $87(2.0)$ & \\
Bleeding (\%) & & & \\
Absent & $52(94.3)$ & $4065(95.4)$ & \\
Present & $5(5.7)$ & $194(4.6)$ & \\
\hline
\end{tabular}

$E G C$ early gastric cancer, $W D$ well differentiated, $M D$ moderately differentiated
Table 3 Short-term outcomes of endoscopic submucosal dissection for papillary versus well- or moderately differentiated early gastric cancer confined to mucosa

\begin{tabular}{|c|c|c|c|}
\hline & \multicolumn{2}{|c|}{ Mucosal cancer } & \multirow[t]{2}{*}{$P$ value } \\
\hline & $\begin{array}{l}\text { Papil- } \\
\text { lary EGC } \\
(n=51)\end{array}$ & $\begin{array}{l}\text { WD or } \\
\text { MD EGC } \\
(n=3527)\end{array}$ & \\
\hline En bloc resection (\%) & & & 0.060 \\
\hline Yes & $48(94.1)$ & $3467(98.3)$ & \\
\hline No & $3(5.9)$ & $60(1.7)$ & \\
\hline $\mathrm{R} 0$ resection (\%) & & & 0.110 \\
\hline Yes & $47(92.2)$ & $3402(96.5)$ & \\
\hline No or undetermined & $4(7.8)$ & $125(3.5)$ & \\
\hline En bloc and $\mathrm{R} 0$ resection (\%) & & & 0.026 \\
\hline Yes & $45(88.2)$ & 3371 (95.6) & \\
\hline No or undetermined & $6(11.8)$ & $156(4.4)$ & \\
\hline Curative resection (\%) & & & $<0.001$ \\
\hline Yes & $37(72.5)$ & 3305 (93.7) & \\
\hline No & $14(27.5)$ & $222(6.3)$ & \\
\hline Lymphatic invasion (\%) & & & $<0.001$ \\
\hline Absent & $43(84.3)$ & $3457(98.0)$ & \\
\hline Present & $8(15.7)$ & $70(2.0)$ & \\
\hline Venous invasion (\%) & & & 0.042 \\
\hline Absent & $50(98.0)$ & $3525(99.9)$ & \\
\hline Present & $1(2.0)$ & $2(0.1)$ & \\
\hline Perforation (\%) & & & 0.189 \\
\hline Absent & $49(96.1)$ & $3473(98.5)$ & \\
\hline Present & $2(3.9)$ & $54(1.5)$ & \\
\hline Bleeding (\%) & & & 0.509 \\
\hline Absent & $48(94.1)$ & $3365(95.4)$ & \\
\hline Present & $3(5.9)$ & $162(4.6)$ & \\
\hline
\end{tabular}

$E G C$ early gastric cancer, $W D$ well differentiated, $M D$ moderately differentiated

mucosal cancers, respectively. The curative resection rates were 72.5 and $93.7 \%$ in papillary and WD or MD EGCs confined to the mucosa, respectively. Both rates were significantly lower for papillary mucosal cancers than for WD or MD EGC. The major reasons for non-curative resection in papillary mucosal EGC were lymphatic invasion and lateral resection margin involvement of the tumor. Lymphatic invasion accounted for $57.1 \%$ and lateral margin involvement accounted for $28.6 \%$ of non-curative resection cases in papillary mucosal EGC.

\section{Long-term outcomes of ESD for papillary EGC}

Figure 1 displays the follow-up outcomes of ESD for papillary EGC. Among 76 patients undergoing ESD for papillary EGC from January 2007 to December 2014, 38 patients with 39 EGCs underwent curative ESD, of which 24 tumors met the absolute criteria and 15 tumors met the expanded 


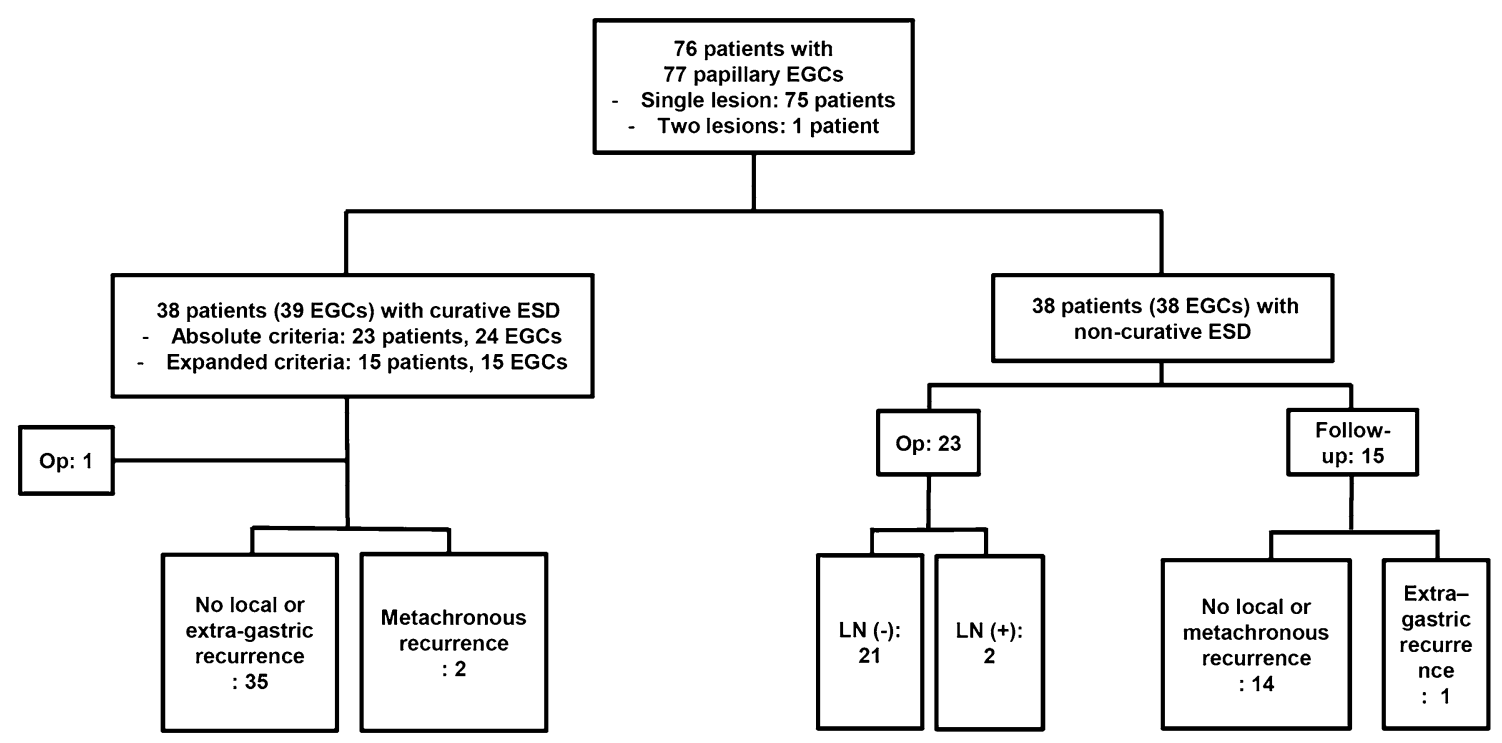

Fig. 1 Flowchart for outcomes of endoscopic submucosal dissection for papillary early gastric cancer. EGC early gastric cancer, ESD endoscopic submucosal dissection, $O p$ operation, $L N$ lymph node

criteria. During a median follow-up period of 58 months (range 2-118 months), two metachronous recurrences $(5.2 \%, 2 / 38)$ were detected, 4 and 9 years after curative ESD, respectively (one case meeting the absolute criteria and one case meeting the expanded criteria). Both patients with metachronous recurrences were curatively treated with ESD and radical gastrectomy. During the follow-up period, no local or extra-gastric recurrences were observed in patients who received curative ESD. One patient whose tumor met the expanded criteria underwent radical gastrectomy after curative ESD. No LN metastasis was found in the surgical specimen of this patient. There was no gastric cancer-related death during the follow-up period after curative ESD for papillary EGC.

\section{Discussion}

In the present study, papillary EGC exhibited more aggressive clinicopathologic features than WD or MD EGC. In addition, the short-term outcomes of ESD for papillary EGC were significantly worse than those for WD or MD EGC. The curative resection rate was only $49.4 \%$ in papillary EGC, while it was $82.2 \%$ in WD or MD EGC. However, once curative resection was achieved, the long-term outcomes were acceptable. After curative ESD for papillary EGC, there was no local or extra-gastric recurrence during a median follow-up period of 58 months. To the best of our knowledge, this is the first long-term follow-up study of ESD for papillary EGC providing evidence for the validity of the current Japanese guidelines.
In the present study, the lymphovascular and submucosal invasion rates were significantly higher for papillary EGC than for WD or MD EGC, consistent with the results of previous studies [2-6]. Interestingly, histological heterogeneity and lateral resection margin involvement of the tumor were found more frequently in papillary EGC than in WD or MD EGC. Adachi et al. [13] reported that papillotubular differentiation was frequently found in the margins of poorly differentiated medullary gastric carcinoma. Based on this result, the authors argued that some of the poorly differentiated medullary carcinomas might arise as papillary adenocarcinoma $[13,14]$. In our previous study on differentiated-type EGC with histological heterogeneity, we observed lateral resection margin involvement more frequently in tumors with histological heterogeneity than in pure differentiatedtype EGCs [15]. In the present study, however, the histological heterogeneity rates did not differ significantly between papillary EGCs with and without lateral margin involvement (22.2 and $15.4 \%$, respectively). Given the aggressive clinicopathologic features of papillary EGC, careful histological evaluation of ESD specimen is required to accurately determine whether curative resection is achieved.

Due to its aggressive tumor behavior, papillary EGC had significantly worse short-term outcomes after ESD than WD or MD EGCs. The curative ESD rates for papillary EGC were $49.4 \%$ in mucosal or submucosal EGC and $72.5 \%$ in mucosal EGC in the present study. These poor short-term outcomes were consistent with the results of a Korean study which reported a curative ESD rate of $45.8 \%$ (11/24) for papillary EGC [3]. Among the two major reasons for noncurative resection in papillary mucosal EGC, the presence of lymphatic invasion could not be accurately assessed before 
ESD. Lateral resection margin involvement was the second most frequent reason for non-curative resection in papillary mucosal EGC. Therefore, four-quadrant mapping biopsies before ESD or wide marking during ESD might be helpful to improve the curative resection rate in papillary EGC.

Despite the poor short-term outcomes, the long-term outcomes of ESD for papillary EGC were favorable once curative resection was achieved. In the present study, no extra-gastric recurrences were observed during a median 58 months of follow-up after curative ESD. Two patients had metachronous recurrences, and both were curatively treated. These favorable results are consistent with our previous report based on large-scale surgical data. No papillary EGC exhibited LN metastasis in the surgical specimen if it met the curative endoscopic resection criteria [7]. A recent Korean study also demonstrated the favorable follow-up outcomes of ESD for papillary EGC: 11 patients underwent curative ESD for papillary EGC, and no recurrences were observed during a median 19 months of follow-up [3].

This study was limited in that it was performed at a single tertiary referral center and had a retrospective design. Second, given the considerable rate of non-curative resection, the cost-effectiveness of ESD for papillary EGC may be questioned, but this was not evaluated in the present study. As the number of papillary EGC patients who underwent curative ESD was limited in this study, further large-scale studies are required to confirm the favorable long-term follow-up outcomes after curative ESD in patients with papillary EGC.

In conclusion, ESD might be indicated for papillary EGC according to the current Japanese guidelines, given the favorable long-term outcomes after curative resection. As papillary EGC has considerable rates of lymphovascular invasion, submucosal invasion, and histological heterogeneity, careful histological examination of ESD specimen is required to accurately determine whether curative resection is achieved.

Author contributions Conception and design: TSK, BHM, and KMK; analysis and interpretation of the data: TSK and BHM; drafting of the article: TSK and BHM; critical revision of the article for important intellectual content: TSK, BHM, KMK, JHL, PLR, and JJK. All the authors listed have approved the final draft submitted.

\section{Compliance with ethical standards}

Conflict of interest The authors declare that they have no conflict of interest.

Human rights statement All procedures followed were in accordance with the ethical standards of the responsible committee on human experimentation (institutional and national) and with the Helsinki Declaration of 1964 and later versions.

Informed consent Informed consent to be included in the study, or the equivalent, was obtained from all patients.

\section{References}

1. Japanese Gastric Cancer A. Japanese gastric cancer treatment guidelines 2014 (ver. 4). Gastric Cancer. 2017;20:1-19.

2. Sekiguchi M, Sekine S, Oda I, Nonaka S, Suzuki H, Yoshinaga $S$, et al. Risk factors for lymphatic and venous involvement in endoscopically resected gastric cancer. J Gastroenterol. 2013;48:706-12.

3. Lee HJ, Kim GH, Park DY, Lee BE, Jeon HK, Jhi JH, et al. Is endoscopic submucosal dissection safe for papillary adenocarcinoma of the stomach? World J Gastroenterol. 2015;21:3944-52.

4. Sekiguchi M, Kushima R, Oda I, Suzuki H, Taniguchi H, Sekine $\mathrm{S}$, et al. Clinical significance of a papillary adenocarcinoma component in early gastric cancer: a single-center retrospective analysis of 628 surgically resected early gastric cancers. J Gastroenterol. 2015;50:424-34.

5. Lee HJ, Kim GH, Park DY, Kim YK, Jeon HK, Lee BE, et al. Endoscopic submucosal dissection for papillary adenocarcinoma of the stomach: is it really safe? Gastric Cancer. 2017;20:978-86.

6. Yu H, Fang C, Chen L, Shi J, Fan X, Zou X, et al. Worse prognosis in papillary, compared to tubular, early gastric carcinoma. $\mathrm{J}$ Cancer. 2017;8:117-23.

7. Min BH, Byeon SJ, Lee JH, Kim KM, An JY, Choi MG, et al. Lymphovascular invasion and lymph node metastasis rates in papillary adenocarcinoma of the stomach: implications for endoscopic resection. Gastric Cancer. 2018;21:680-8.

8. Min BH, Kim ER, Kim KM, Park CK, Lee JH, Rhee PL, et al. Surveillance strategy based on the incidence and patterns of recurrence after curative endoscopic submucosal dissection for early gastric cancer. Endoscopy. 2015;47:784-93.

9. Pyo JH, Lee H, Min BH, Lee JH, Choi MG, Lee JH, et al. Longterm outcome of endoscopic resection vs. surgery for early gastric cancer: a non-inferiority-matched cohort study. Am J Gastroenterol. 2016;111:240-9.

10. Gotoda T, Yamamoto H, Soetikno RM. Endoscopic submucosal dissection of early gastric cancer. J Gastroenterol. 2006;41:929-42.

11. Lauwers GY, Carneiro F, Graham DY, Curado MP, Franceschi S, Montgomery E, et al. Gastric carcinoma. In: Bosman FT, Carneiro F, Hruban RH, Theise ND, editors. WHO classification of tumours of the digestive system. Lyon: IARC; 2010. pp. 48-58.

12. Kim WH, Park CK, Kim YB, Kim YW, Kim HG, Bae HI. A standardized pathology report for gastric cancer. Korean J Pathol. 2005;39:106-13.

13. Adachi Y, Mori M, Maehara Y, Sugimachi K. Poorly differentiated medullary carcinoma of the stomach. Cancer. 1992;70:1462-6.

14. Yasuda K, Adachi Y, Shiraishi N, Maeo S, Kitano S. Papillary adenocarcinoma of the stomach. Gastric Cancer. 2000;3:33-8.

15. Min BH, Kim KM, Park CK, Lee JH, Rhee PL, Rhee JC, et al. Outcomes of endoscopic submucosal dissection for differentiatedtype early gastric cancer with histological heterogeneity. Gastric Cancer. 2015;18:618-26. 